\title{
Asociación entre concentraciones séricas de lactato deshidrogenasa en las primeras 12 horas de vida y encefalopatía hipóxico-isquémica. Estudio preliminar
}

\author{
Association between serum lactate dehydrogenase levels in \\ the first 12 hours of life and the development of hypoxic- \\ ischemic encephalopathy. A preliminary study
}

Sergio Alfonso Patrón-Chí, ${ }^{1}$ Enrique Eduardo Peraza-López, ${ }^{2}$ Alicia Leonor Zapata-Peraza ${ }^{3}$

\begin{abstract}
Resumen
INTRODUCCIÓN: La encefalopatía hipóxico-isquémica es la consecuencia más grave de la asfixia perinatal. Las variaciones en las concentraciones séricas de algunas enzimas intracelulares durante las primeras horas de vida extrauterina podrían ser útiles como marcadores de riesgo de encefalopatía hipóxico-isquémica.

OBJETIVO: Analizar la posible asociación entre el cambio en los valores séricos de lactato deshidrogenasa con respecto a distintos intervalos en las primeras 12 horas de vida extrauterina y la encefalopatía hipóxico-isquémica en recién nacidos con factores de riesgo para asfixia neonatal.

MATERIALES Y MÉTODOS: Estudio de prueba diagnóstica. Se efectuaron determinaciones de lactato deshidrogenasa a las 0,6 y 12 horas de vida extrauterina en neonatos con diagnóstico clínico de asfixia perinatal $(n=10)$ y en pacientes con factores de riesgo que no cumplieron los criterios diagnósticos $(n=10)$.

RESULTADOS: El punto de corte para lactato deshidrogenasa sérica se estableció en 775 UI/L, con sensibilidad de $90 \%$ y especificidad de $60 \%$ para encefalopatía hipóxicoisquémica. Un incremento en los valores séricos de lactato deshidrogenasa igual o superior a $0.33 \%$ /hora entre las horas 0 y 12 tuvo sensibilidad de $90 \%$ y especificidad de $100 \%$ respecto del inicio de la encefalopatía hipóxico-isquémica.

CONCLUSIÓN: La variación en las concentraciones séricas de lactato deshidrogenasa en las primeras 12 horas de vida se asocia con encefalopatía hipóxico-isquémica.

PALABRAS CLAVE: Encefalopatía hipóxico-isquémica; asfixia perinatal; lactato deshidrogenasa; asfixia.

Abstract

INTRODUCTION: Hypoxic-ischemic encephalopathy is the most severe consequence of perinatal asphyxia. The rate of change in the serum levels of some intracellular enzymes among the first hours of extrauterine life may serve as indication of heighten risk for the development of Hypoxic-ischemic encephalopathy.

OBJECTIVE: To analyze the possible association between the rates of change in serum lactate dehydrogenase values in the first 12 hours of extrauterine life and the development of hypoxic-ischemic encephalopathy.

METHODS: Diagnostic test study. Lactate dehydrogenase determinations were performed at 0,6 and 12 hours of extrauterine life in neonates who presented clinical diagnosis of perinatal asphyxia $(n=10)$ and in patients with risk factors that did not meet asphyxia criteria $(n=10)$.
\end{abstract}

\footnotetext{
${ }^{1}$ Médico cirujano, especialidad en Pediatría.

${ }^{2}$ Médico cirujano, egresado de la Universidad Autónoma de Yucatán.

${ }^{3}$ Médico cirujano, investigadora del Centro de investigaciones Regionales Dr Hideyo Noguchi.
}

Universidad Autónoma de Yucatán, México.

Recibido: 14 de mayo 2017

Aceptado: 14 de mayo 2018

Correspondencia

Enrique Eduardo Peraza López

E_peraza_l@hotmail.com

Este artículo debe citarse como Patrón-Chí SA, Peraza-López EE, ZapataPeraza AL. Asociación entre concentraciones séricas de lactato deshidrogenasa en las primeras 12 horas de vida y encefalopatía hipóxico-isquémica. Estudio preliminar. Acta Pediatr Mex. 2018;39(5):271-282. 
RESULTS: Setting a cut-off point for serum lactate dehydrogenase at $775 \mathrm{IU} / \mathrm{L}$, a sensitivity of $90 \%$ and specificity of $60 \%$ for the development of hypoxic-ischemic encephalopathy was obtained. An increase for serum values of lactate dehydrogenase equal to or greater than $0.33 \%$ /hour between 0 and 12 hours showed sensitivity of $90 \%$ and specificity $100 \%$ with respect to the development of hypoxic-ischemic encephalopathy. CONCLUSION: Variation in serum lactate dehydrogenase levels in the first 12 hours of life is associated with the development of hypoxic-ischemic encephalopathy.

KEY WORDS: Hypoxic-ischemic encephalopathy; Perinatal asphyxia; Lactate dehydrogenase; Asphyxia.

\section{INTRODUCCIÓN}

La asfixia neonatal es un síndrome clínico de etiología multifactorial cuya característica definitoria es la suspensión (o grave disminución) de la capacidad de intercambio gaseoso del feto durante el periparto. Las alteraciones que condicionan este síndrome pueden ocurrir en la placenta o en los pulmones del recién nacido. Para que sobrevenga la asfixia neonatal la disminución del intercambio gaseoso debe ocasionar hipoxemia, hipercapnia y lesión celular por hipoxia. ${ }^{1,2}$

Según el Colegio Americano de Ginecobstetricia se requiere la coexistencia de al menos una de las siguientes condiciones para establecer el diagnóstico de asfixia neonatal: $\mathrm{pH}$ en sangre de la arteria umbilical inferior a 7; déficit de base en sangre de la arteria umbilical igual o mayor a 12 $\mathrm{mEq} / \mathrm{L} ;$ puntaje Apgar $\leq 5$ a los 5 minutos de vida extrauterina; datos de insuficiencia multiorgánica, y datos de encefalopatía hipóxico-isquémica moderada a grave en las primeras horas de vida. ${ }^{2}$

La encefalopatía hipóxico-isquémica es la consecuencia más grave de la asfixia neonatal. Constituye un síndrome clínico cuya característica definitoria es la perturbación de la función neurológica manifestada durante los primeros días de vida extrauterina del infante de término.
Las alteraciones neurológicas en la encefalopatía hipóxico-isquémica se manifiestan como dificultad para iniciar o mantener la respiración, depresión en el tono muscular y reflejos tendinosos, disminución en el nivel de consciencia $y$, frecuentemente, convulsiones. ${ }^{2,3}$

En el mundo ocurren alrededor de 130 millones de nacimientos al año. Del total de nacidos vivos, alrededor de 4 millones mueren en los primeros 28 días de vida extrauterina. En el ámbito mundial, la asfixia neonatal es considerada la tercera causa de mortalidad en el periodo neonatal $(23 \%)$, solo por detrás de la prematurez $(28 \%)$ y de las causas infecciosas (26\%). El 99\% de estas defunciones ocurre en países de medianos a bajos ingresos (entre ellos México). ${ }^{4-6}$

De acuerdo con la Organización Mundial de la Salud, cada año entre 4 y 9 millones de neonatos cumplen con criterios para el diagnóstico de asfixia neonatal; de éstos, 1.2 millones fallecen a consecuencia directa de la asfixia neonatal y una cantidad semejante tendrá consecuencias adversas a largo plazo. Entre las consecuencias de la asfixia neonatal destacan: epilepsia, parálisis cerebral o retraso en el desarrollo psicomotor. ${ }^{7,8}$ La asfixia neonatal moderada a grave origina una mortalidad de $24 \%$ y un riesgo de discapacidad cognitiva en los supervivientes de $78 \%{ }^{6}$ 
En México, 60\% de la mortalidad en menores de un año ocurre en el periodo neonatal. ${ }^{9} \mathrm{La}$ asfixia neonatal es una de las principales causas de mortalidad durante el primer mes de vida extrauterina en México (se relacionó con 49.4\% de las defunciones en 2003). ${ }^{10,11}$

El diagnóstico temprano de la asfixia neonatal se basa en datos clínicos, de laboratorio y en parámetros gasométricos obtenidos a partir de sangre arterial del cordón umbilical. Muchos de los pacientes con evento asfíctico nacen fuera de los centros de salud que cuentan con los recursos necesarios para establecer tempranamente el diagnóstico; la falta de acceso oportuno a una gasometría en sangre arterial umbilical es una de las limitaciones más frecuentes. ${ }^{10,11}$

En forma aislada, los parámetros clínicos al momento del nacimiento tienen una correlación limitada con el daño neurológico secundario a la asfixia perinatal (hasta $51 \%$ de los lactantes que experimentaron algún grado de encefalopatía reciben una puntuación de Apgar de 4 o más a los 5 minutos de vida extrauterina). ${ }^{12}$ Esto cobra especial relevancia en una subpoblación de neonatos con "eventos centinela" durante el periparto; es decir, situaciones que se han vinculado con un riesgo incrementado de asfixia neonatal. ${ }^{13}$

En cualquier situación en la que ocurra un daño celular importante (entre ellos la hipoxiaisquemia) habrá perturbaciones en la membrana celular con la consecuente liberación de enzimas intracelulares hacia la circulación sistémica. La detección y cuantificación de estas sustancias puede utilizarse como un marcador de daño tisular. Posterior a un evento asfíctico neonatal se ha reportado elevación de las concentraciones séricas de aminotransferasa de alanina, aminotransferasa de aspartato y lactato deshidrogenasa. ${ }^{14-16}$
En estudios previos, la elevación de la aminotransferasa de alanina en el recién nacido ha demostrado una alta especificidad para predecir el riesgo de encefalopatía hipóxico-isquémica, mientras que un incremento en la lactato deshidrogenasa resulta un marcador altamente sensible para daño celular. ${ }^{15-17}$

El incremento en las concentraciones séricas de estas enzimas, así como sus patrones diferenciados de aclaramiento en plasma, pueden servir como marcadores predictivos de encefalopatía hipóxico-isquémica en neonatos con signos de sufrimiento fetal en el periparto., ${ }^{1,15-17}$

La importancia de establecer el diagnóstico oportuno de la asfixia neonatal radica en la posibilidad de efectuar ciertos procedimientos terapéuticos que reducen la probabilidad de que el neonato tenga secuelas neurológicas, en particular la hipotermia terapéutica; sin embargo, las ventajas neuroprotectoras de esta medida disminuyen rápidamente si se retrasa su aplicación, solo habiéndose demostrado ventaja si se inicia en las 6 horas posteriores al evento de asfixia. ${ }^{17-23}$

El objetivo del estudio fue: analizar la posible asociación entre el cambio en los valores séricos de lactato deshidrogenasa con respecto a distintos intervalos en las primeras 12 horas de vida extrauterina y la encefalopatía hipóxicoisquémica en recién nacidos con factores de riesgo para asfixia neonatal.

\section{MATERIALES Y MÉTODOS}

Estudio de prueba diagnóstica efectuado de febrero a septiembre de 2016 en el área de Pediatría de nuestra institución. Se consideró como hipótesis alternativa: "En neonatos con evento centinela la variación en las concentraciones séricas de lactato deshidrogenasa durante las primeras 12 horas de vida extrauterina se asocia 
con el diagnóstico de asfixia neonatal y encefalopatía hipóxico-isquémica".

Se entiende por evento centinela cualquiera de las siguientes circunstancias: prolapso o circular de cordón umbilical, desprendimiento prematuro de placenta normoinserta, meconio en líquido amniótico, bradicardia fetal, distocia de hombros o necesidad de extracción instrumental [vaccum o fórceps].
Se estudiaron 20 neonatos que tuvieron algún evento centinela para asfixia neonatal. Los casos $(n=10)$ tuvieron, además, alguno de los datos clínicos o gasométricos recomendados por el Colegio Americano de Ginecoobstetricia para el diagnóstico de asfixia neonatal; los controles $(n=10)$ solo tuvieron el evento centinela. Los criterios de inclusión, exclusión y eliminación, así como los procedimientos efectuados se ilustran en la Figura 1.
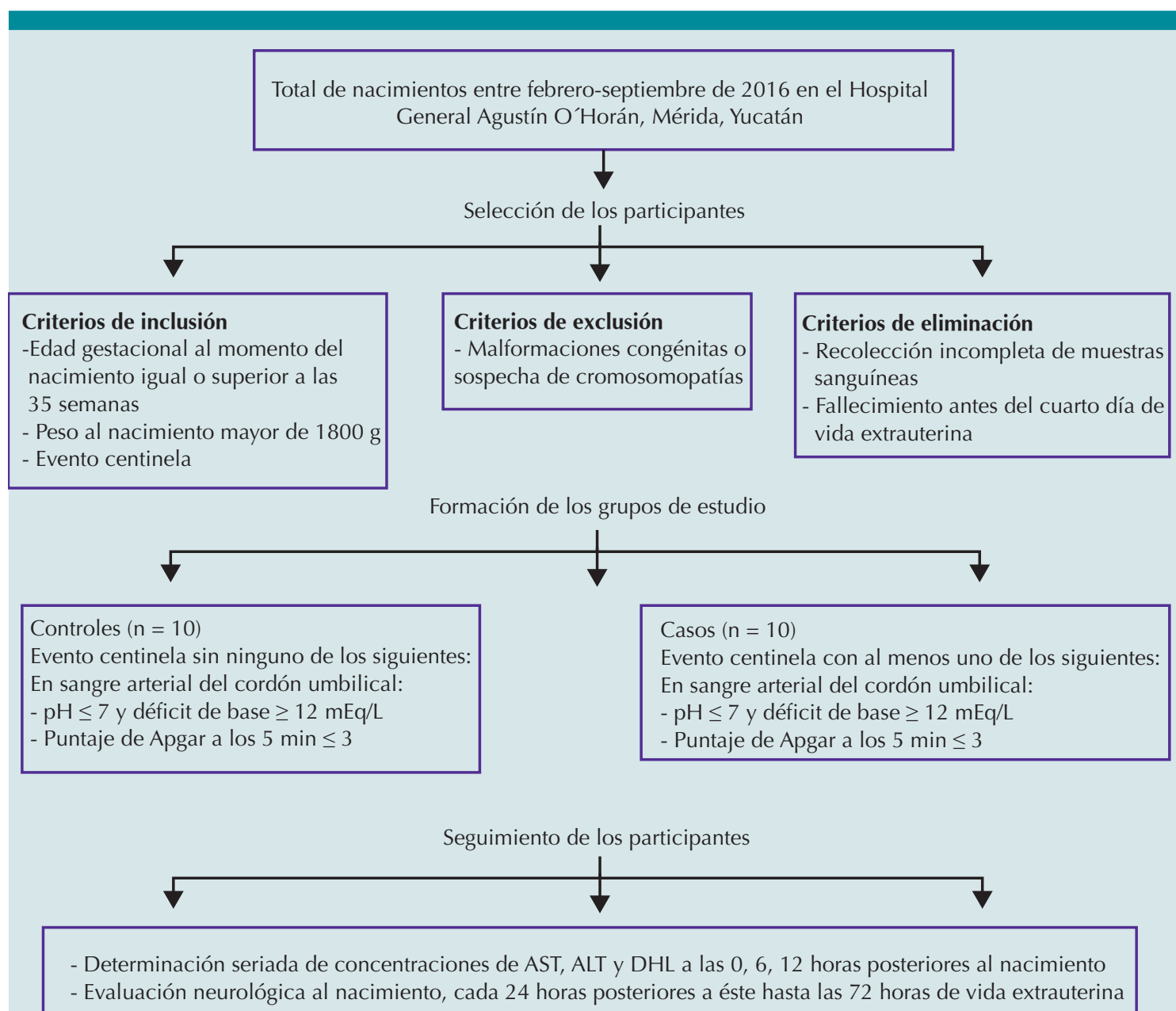

Figura 1. Criterios de inclusión, exclusión y eliminación, visión general de los procedimientos efectuados en el estudio.

AST: aminotransferasa de aspartato. ALT: aminotransferasa de alanina. LDH: lactato deshidrogenasa (DHL) 
Se definió como caso de estudio al paciente con al menos un evento centinela y que al momento del nacimiento manifestó, al menos, uno de los siguientes parámetros: gasometría arterial umbilical con $\mathrm{pH} \leq 7$ o déficit de base $\leq 12 \mathrm{mEq} / \mathrm{L}$, puntaje en escala de Apgar $\leq 3$ a los 5 minutos de vida extrauterina o alteraciones neurológicas o insuficiencia orgánica múltiple en las primeras 24 horas de vida extrauterina.

\section{Procedimientos}

Para la obtención de la gasometría arterial se utilizó una jeringa estéril de $1 \mathrm{~mL}$ que se impregnó con $0.01 \mathrm{~mL}$ de heparina sódica; se recolectaron, al menos, 0.4 cc de sangre arterial del cordón umbilical procedente de cada uno de los participantes. Las pruebas se procesaron inmediatamente con un gasómetro "Stat Phox Plus L" de la marca Prodimol. Se registraron los valores de $\mathrm{pH}$, lactato y exceso de base para análisis posterior. Para la determinación de los valores de las enzimas séricas se recolectaron 400 microlitros de sangre venosa a las 0,6 y 12 horas. Las muestras se colocaron en tubos BD Microtainer para suero y se procesaron de acuerdo con el protocolo estandarizado del laboratorio químico de nuestra institución.

Además de la medición de la lactato deshidrogenasa se registraron las concentraciones de otros marcadores séricos asociados con asfixia neonatal: ${ }^{14-6}$ lactato, exceso de base, AST y ALT. Se tomaron como límites de referencia para la normalidad en las primeras 24 horas de vida extrauterina: AST 47-150 UI/L, ALT 13-45 UI/L y lactato deshidrogenasa 290-775 UI/L.

En relación con la encefalopatía hipóxico-isquémica se realizaron evaluaciones neurológicas seriadas a cada uno de los participantes durante los 4 primeros días de vida extrauterina. Se siguieron los criterios de Sarnat para el diagnóstico y estatificación de la encefalopatía hipóxico- isquémica; se evaluaron: estado de consciencia, tono muscular, succión, esfuerzo ventilatorio, reflejos primitivos y convulsiones.

El procesamiento de la información se efectuó mediante el paquete estadístico SPSS v.23. Durante el estudio se utilizó como patrón de referencia la definición de encefalopatía hipóxico-isquémica de los criterios de Sarnat.

Se determinaron las medidas de tendencia central para cada variable estudiada. Como prueba de contraste de hipótesis se empleó la prueba exacta de Fisher y el coeficiente de correlación de Spearman para datos cuantitativos. Para la determinación de los puntos de corte óptimos para la variación en las concentraciones de lactato deshidrogenasa, con respecto al tiempo, se emplearon el índice de Youden y curvas ROC. Se calculó la sensibilidad, especificidad, valores predictivos positivos y negativos de los puntos de corte seleccionados.

El protocolo de esta investigación fue aprobado por el Comité de Ética de nuestra institución. Este estudio se basó en las normas éticas de obligación universal para la investigación en humanos, adoptadas por la Secretaría de Salud e indicadas en el Título V, Artículos del 96 al 103 de la Ley General de Salud de los Estados Unidos Mexicanos y en las normas relativas a la Ética de la Investigación Biomédica en Humanos fijadas en la Declaración de Helsinki y modificadas en la Asamblea Médica Mundial de Hong Kong. Se solicitó el consentimiento informado del responsable legal de cada participante, mismo que fue otorgado. Los datos reunidos y utilizados en esta investigación se procesaron, siempre, de manera anónima.

\section{RESULTADOS}

El punto de corte para lactato deshidrogenasa sérica se estableció en 775 UI/L, con sensibilidad 
de $90 \%$ y especificidad de $60 \%$ para encefalopatía hipóxico-isquémica. No se encontraron diferencias estadísticamente significativas entre el grupo de casos y el de controles con respecto a sexo de los participantes, edad gestacional o vía de nacimiento.

Todos los casos padecieron algún grado de encefalopatía, no se detectaron alteraciones neurológicas en ninguno de los controles. En relación con el puntaje Apgar a los 5 minutos de vida extrauterina se observó una diferencia estadísticamente significativa; los casos tuvieron una media de 4.5 respecto a una media de 8.1 en los controles $(p<0.001)$. El $60 \%$ de los casos tuvo Apgar de 4 o superior a los 5 minutos de vida extrauterina respecto del $100 \%$ de los controles. Cuadro 1

Los valores séricos de lactato deshidrogenasa de casos y controles medidos a las 0,6 y 12 horas de vida extrauterina tuvieron una diferencia estadísticamente significativa, siempre con resultado superior en los casos $(p<.001)$.
Las concentraciones séricas de lactato tuvieron un comportamiento similar al descrito para la lactato deshidrogenasa. Los casos tuvieron una media de $7.09 \mathrm{mmol} / \mathrm{L}$ respecto de los controles, quienes reportaron una media de $1.77 \mathrm{mmol} / \mathrm{L}$ $(p<0.001)$. Se obtuvo el mismo grado de significación al comparar el déficit de base entre casos ( media $=-17.85 \mathrm{mEq} / \mathrm{L}$ ) y controles (media $=-5.64$ $\mathrm{mEq} / \mathrm{L})(\mathrm{p}<.001)$. No se encontraron diferencias significativas entre casos y controles respecto de los valores de aspartato aminotransferasa $(p>0.1)$.

Se evaluó el valor sérico máximo de lactato deshidrogenasa, AST, ALT alcanzado por cada participante durante el estudio con respecto al límite superior del intervalo de referencia. Se empleó un punto de corte de $775 \mathrm{UI} / \mathrm{L}$ para la lactato deshidrogenasa, con sensibilidad de $90 \%$ y especificidad de $60 \%$ para el diagnóstico de encefalopatía hipóxico-isquémica ( $p=$ 0.02). Al evaluar las concentraciones máximas registradas de ALT y AST valoradas con puntos de corte de $45 \mathrm{UI} / \mathrm{L}$ y $150 \mathrm{UI}$, respectivamente, no se encontraron diferencias significativas entre casos y controles.

Cuadro 1. Características demográficas y clínicas de casos y controles

\begin{tabular}{|c|c|c|c|c|c|}
\hline Variable (dicotómica) & Grupo & Media (DE) & Intervalo & Total & Significación \\
\hline \multirow{2}{*}{ Sexo } & Casos & Masculino $=5$ & Femenino $=5$ & $\mathrm{n}=10$ & \multirow{2}{*}{$p=0.5$} \\
\hline & Controles & Masculino $=6$ & Femenino $=4$ & $\mathrm{n}=10$ & \\
\hline \multirow{2}{*}{ Vía de nacimiento } & Casos & Vaginal = 2 & Abdominal $=8$ & $\mathrm{n}=10$ & \multirow{2}{*}{$p=0.79$} \\
\hline & Controles & Vaginal $=2$ & Abdominal $=8$ & $\mathrm{n}=10$ & \\
\hline \multirow{2}{*}{ Encefalopatía } & Casos & $\mathrm{Si}=10$ & No $=0$ & $\mathrm{n}=10$ & \multirow{2}{*}{$\mathrm{p}<0.001$} \\
\hline & Controles & $\mathrm{Si}=0$ & $\mathrm{No}=10$ & $\mathrm{n}=10$ & \\
\hline $\begin{array}{l}\text { Variable } \\
\text { (cuantitativa) }\end{array}$ & Grupo & Media (DE) & Intervalo & Rango & Significación \\
\hline \multirow{2}{*}{ Semanas de gestación } & Casos & $37.1(2.13)$ & $35-40$ & 5 & \multirow{2}{*}{$p=0.6$} \\
\hline & Controles & $37.8(2.35)$ & $34-42$ & 7 & \\
\hline \multirow{2}{*}{ Puntaje Apgar } & Casos & $4.5(2.5)$ & $0-8$ & 8 & \multirow{2}{*}{$\mathrm{p}<0.001$} \\
\hline & Controles & $8.1(0.8756)$ & $6-9$ & 3 & \\
\hline pH arterial umbilical & Casos & $6.76(0.23)$ & $6.2-6.96$ & 0.76 & $p<0.001$ \\
\hline
\end{tabular}


El patrón de cambio en las concentraciones séricas de lactato deshidrogenasa con respecto al tiempo tuvo una diferencia estadísticamente significativa entre casos y controles. En los pacientes con encefalopatía hipóxico-isquémica las concentraciones séricas de lactato deshidrogenasa tuvieron un incremento progresivo durante las primeras 12 horas de vida extrauterina, mientras que en el caso de los controles los valores iniciales tendieron a disminuir con el tiempo ( $p<0.001$ al comparar hora 0 y 12 de vida extrauterina). Cuadro 2
Para determinar el punto de corte óptimo en relación con la variación en las concentraciones de lactato deshidrogenasa, con respecto al tiempo de vida extrauterina, se empleó el índice de Youden (sensibilidad + especificidad -1) y curvas ROC. En esta serie se determinó como punto de corte óptimo un incremento en las concentraciones séricas de lactato deshidrogenasa de al menos $0.33 \%$ por hora respecto de la LDH basal de cada participante.

Con el punto de corte descrito y con base en el cálculo del cambio porcentual entre las con-

Cuadro 2. Media de los valores enzimáticos medidos en los participantes

\begin{tabular}{|c|c|c|c|c|c|}
\hline Variable & Grupo & Media (DE) & Intervalo & Rango & Significación \\
\hline \multirow{2}{*}{$\begin{array}{l}\mathrm{LDH} \\
0 \text { horas de vida } \\
\text { extrauterina (UI/L) }\end{array}$} & Casos & $926.3(218)$ & $721-1454$ & 733 & \multirow[b]{2}{*}{$p<0.05$} \\
\hline & Controles & $676.7(223.6)$ & $322-1023$ & 701 & \\
\hline \multirow{2}{*}{$\begin{array}{l}\text { LDH } \\
6 \text { horas de vida } \\
\text { extrauterina (UI/L) }\end{array}$} & Casos & $1119.7(360)$ & $549-1754$ & 1205 & \multirow{2}{*}{$p<0.01$} \\
\hline & Controles & $543.4(219.3)$ & $333-921$ & 588 & \\
\hline \multirow{2}{*}{$\begin{array}{l}\text { LDH } \\
12 \text { horas de vida } \\
\text { extrauterina (UI/L) }\end{array}$} & Casos & $1370.9(503)$ & $608-2081$ & 1473 & \multirow[b]{2}{*}{$\mathrm{p}<0.01$} \\
\hline & Controles & $472.5(216)$ & $234-822$ & 588 & \\
\hline \multirow{2}{*}{$\begin{array}{l}\text { ALT } \\
0 \text { horas de vida } \\
\text { extrauterina (UI/L) }\end{array}$} & Casos & $27.8(24.5)$ & $3-65$ & 62 & \multirow[b]{2}{*}{$p=0.83$} \\
\hline & Controles & $26(11.94)$ & $7-45$ & 38 & \\
\hline \multirow{2}{*}{$\begin{array}{l}\text { ALT } \\
6 \text { horas de vida } \\
\text { extrauterina }(\mathrm{UI} / \mathrm{L})\end{array}$} & Casos & $39.7(21)$ & $9-78$ & 69 & \multirow[b]{2}{*}{$p=0.74$} \\
\hline & Controles & $36.8(19.72)$ & $7-77$ & 70 & \\
\hline \multirow{2}{*}{$\begin{array}{l}\text { ALT } \\
12 \text { horas de vida } \\
\text { extrauterina (UI/L) }\end{array}$} & Casos & $40.1(21.4)$ & $9-85$ & 76 & \multirow{2}{*}{$p=0.64$} \\
\hline & Controles & $36.2(16.4)$ & $17-67$ & 50 & \\
\hline \multirow{2}{*}{$\begin{array}{l}\text { AST } \\
0 \text { horas de vida } \\
\text { extrauterina }(\mathrm{UI} / \mathrm{L})\end{array}$} & Casos & $122(91)$ & $7-345$ & 338 & \multirow[b]{2}{*}{$p=0.78$} \\
\hline & Controles & $113.3(44)$ & $53-223$ & 170 & \\
\hline \multirow{2}{*}{$\begin{array}{l}\text { AST } \\
\text { Horas de vida } \\
\text { extrauterina (UI/L) }\end{array}$} & Casos & 118(101.7) & $33-371$ & 338 & \multirow{2}{*}{$p=0.56$} \\
\hline & Controles & $99.4(32)$ & 23-152 & 129 & \\
\hline \multirow{2}{*}{$\begin{array}{l}\text { AST } \\
12 \text { horas }(\mathrm{UI} / \mathrm{L})\end{array}$} & Casos & $127.8(111.3)$ & $34-402$ & 368 & \multirow{2}{*}{$p=0.23$} \\
\hline & Controles & $83.6(32)$ & $22-142$ & 120 & \\
\hline \multirow{2}{*}{$\begin{array}{l}\text { Lactato } \\
0 \text { horas de vida } \\
\text { extrauterina }(\mathrm{mmol} / \mathrm{L})\end{array}$} & Casos & $7.09(3.67)$ & $3.3-15.3$ & 12 & \multirow[b]{2}{*}{$p<0.01$} \\
\hline & Controles & $1.77(0.51)$ & $1.0-2.3$ & 1.3 & \\
\hline \multirow{2}{*}{$\begin{array}{l}\text { Déficit de base } \\
0 \text { horas de vida } \\
\text { extrauterina }(\mathrm{mEq} / \mathrm{L})\end{array}$} & Casos & $-17.85(4.56)$ & $-26.9--17.85$ & 14.9 & \multirow[b]{2}{*}{$\mathrm{p}<0.01$} \\
\hline & Controles & $-5.64(2.62)$ & $-9--1$ & 8 & \\
\hline
\end{tabular}

AST: aminotransferasa de aspartato; ALT: aminotransferasa de alanina; LDH: lactato deshidrogenasa; DE: desviación estándar 
centraciones séricas de lactato deshidrogenasa al nacimiento y a las 12 horas se obtuvo una sensibilidad de $90 \%$, especificidad de $100 \%$, valor predictivo positivo de $100 \%$ y valor predictivo negativo de $90.9 \%$. En intervalos menores (0-6 y 6-12 horas de vida extrauterina) se encontró menor sensibilidad y especificidad para este punto de corte; sin embargo, la asociación descrita sigue teniendo significación estadística. Figura 2

\section{y Cuadro 3}

\section{DISCUSIÓN}

Se encontró asociación estadísticamente significativa entre las concentraciones séricas máximas de lactato deshidrogenasa registradas durante las primeras 12 horas de vida extrauterina y la encefalopatía hipóxico-isquémica. Este resultado coincide con lo descrito por Karlsson en $2010,{ }^{14}$ quien concluyó que las concentraciones de lactato deshidrogenasa sérica en neonatos con probable evento asfíctico constituyen un marcador poco específico, pero altamente sensible del daño orgánico ocasionado por la hipoxia-isquemia. Karlsson ${ }^{14}$ identificó una diferencia estadísticamente significativa entre las concentraciones de lactato deshidrogenasa entre pacientes con encefalopatía hipóxico-isquémica y los controles (media casos encefalopatía hipóxico-isquémica $2076 \mathrm{UI} / \mathrm{I}$, controles 575

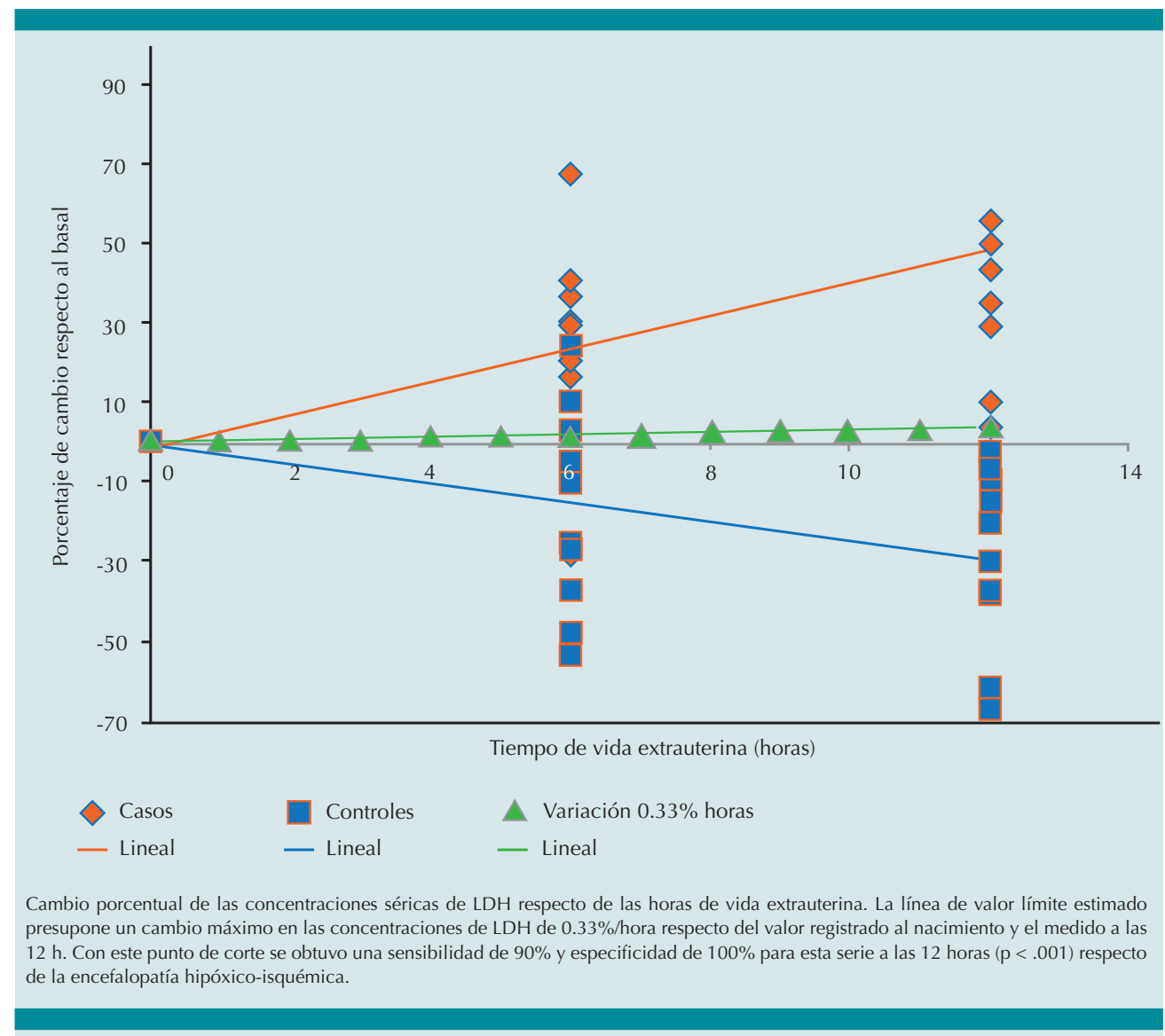

Figura 2. Variación porcentual en las concentraciones de LDH respecto del tiempo durante las primeras 12 horas de vida extrauterina. 
Patrón-Chí et al. Concentraciones de lactato deshidrogenasa y encefalopatía hipóxico-isquémica neonatal

Cuadro 3. Evaluación de los valores enzimáticos en el suero de los participantes respecto de los puntos de corte evaluados

\begin{tabular}{|c|c|c|c|c|c|c|c|c|c|c|}
\hline Variable & $\begin{array}{l}\text { Punto } \\
\text { de corte }\end{array}$ & VP & FP & VN & FN & $\mathbf{S}$ & E & VPP & VPN & Significación \\
\hline LDH (UI/L) & $775 \mathrm{UI} / \mathrm{L}$ & 9 & 4 & 6 & 1 & 90 & 60 & 69 & 85.7 & $p=0.02$ \\
\hline ALT (UI/L) & $45 \mathrm{UI} / \mathrm{L}$ & 5 & 4 & 6 & 5 & 50 & 60 & 55.5 & 54 & $p=0.5$ \\
\hline AST (UI/L) & $150 \mathrm{UI} / \mathrm{L}$ & 3 & 2 & 8 & 7 & 30 & 80 & 60 & 53 & $p=0.2$ \\
\hline $\begin{array}{l}\text { Variación LDH horas 0-6 } \\
\text { (porcentaje/hora) }\end{array}$ & $\begin{array}{c}0.33 \% / \\
\text { hora }\end{array}$ & 6 & 1 & 9 & 4 & 60 & 90 & 85.71 & 69.23 & $p=0.018$ \\
\hline $\begin{array}{l}\text { Variación LDH horas 6-12 } \\
\text { (porcentaje/hora) }\end{array}$ & $\begin{array}{c}0.33 \% / \\
\text { hora }\end{array}$ & 7 & 2 & 8 & 3 & 70 & 80 & 77.7 & 72.72 & $p=0.018$ \\
\hline $\begin{array}{l}\text { Variación LDH horas 0-12 } \\
\text { (porcentaje/hora) }\end{array}$ & $\begin{array}{c}0.33 \% / \\
\text { hora }\end{array}$ & 9 & 0 & 10 & 1 & 90 & 100 & 100 & $90.9 \%$ & $p=<0.001$ \\
\hline
\end{tabular}

VP: verdaderos positivos; FP: falsos positivos; FN: falsos negativos; VN: verdaderos negativos; S: sensibilidad; E: especificidad; VPP: valor predictivo positivo; VPN: valor predictivo negativo; AST: aminotransferasa de aspartato; ALT: aminotransferasa de alanina; LDH: lactato deshidrogenasa.

$\mathrm{UI} / \mathrm{L})$, similar a la diferencia observada en nuestro estudio (1370.9 UI/L en casos, $472 \mathrm{UI} / \mathrm{dl}$ en controles a las 12 horas de vida extrauterina). Karlsson también identificó a la ALT como un marcador sensible y específico en relación con la asfixia perinatal (media de casos de encefalopatía hipóxico-isquémica $49 \mathrm{UI} / \mathrm{l}$, controles $10 \mathrm{UI} / \mathrm{l}$, punto de corte $15 \mathrm{u} / \mathrm{L}$ ), hallazgo que no pudo reproducirse en nuestro estudio (ALT en casos $118 \mathrm{UI} / \mathrm{L}$, controles $99.4 \mathrm{UI} / \mathrm{L}$ a las 6 horas de vida extrauterina $p=0.79) .{ }^{14}$

Beken, en 2014, ${ }^{1}$ encontró que los valores de lactato deshidrogenasa tienen mejor sensibilidad y especificidad en relación con el diagnóstico de asfixia neonatal respecto de los valores séricos de creatincinasa total, ácido úrico y lactato. En forma análoga, en este estudio se encontró que las concentraciones de lactato deshidrogenasa (y sobre todo su variación en el tiempo) se correlacionan con encefalopatía hipóxico-isquémica.

La concentración de lactato en sangre arterial umbilical demostró una alta sensibilidad y especificidad en esta serie. Con un punto de corte de $3.3 \mathrm{mmol} / \mathrm{L}$ de lactato se obtuvo una sensibilidad y especificidad de $100 \%$ para encefalopatía hipóxico-isquémica. Esto es similar a lo descrito por Cruz en 2012, quien identificó al lactato en sangre arterial umbilical como un marcador útil en la predicción de encefalopatía hipóxico-isquémica. En su estudio, encontró que un punto de corte de $4 \mathrm{mmol} / \mathrm{L}$ ofrecía una sensibilidad y especificidad del $100 \%$ para alteraciones neurológicas (sin tomar en cuenta el grado de la encefalopatía). ${ }^{24}$

Este hallazgo se ha repetido en otros estudios, por ejemplo, Willberg en 2010 mediante el análisis de 13,735 gasometrías arteriales umbilicales concluyó que las concentraciones de lactato tenían el mismo valor predictivo para encefalopatía hipóxico-isquémica que el déficit de base. ${ }^{25}$ Beken, en 2014, reportó una correlación entre mayores concentraciones de lactato y lactato deshidrogenasa en suero con mayor grado de encefalopatía hipóxico-isquémica en neonatos con asfixia, hallazgo que no pudo ser replicado en este estudio. ${ }^{1}$

El puntaje Apgar de los pacientes con encefalopatía fluctuó entre 1 y 8 . El $60 \%$ de los 
participantes con encefalopatía hipóxico-isquémica tuvo Apgar de 4 o superior. Esto coincide con lo descrito por Nelsson en 2012, quien en una serie con 4165 neonatos con diagnóstico de probable encefalopatía hipóxico-isquémica encontró que solo $49.4 \%$ de los participantes recibió una calificación de Apgar $\leq 3$ a los 5 minutos de vida extrauterina. ${ }^{12}$

En nuestro estudio fue posible demostrar una asociación entre la variación en las concentraciones séricas de lactato deshidrogenasa con respecto al tiempo de vida extrauterina y la encefalopatía hipóxico-isquémica.

Este hallazgo se explica debido al patrón de muerte celular bimodal que ocurre posterior a una lesión debida a hipoxia-isquemia. Posterior a un evento agudo en el que se restringe el aporte de oxígeno y glucosa en un grado suficiente para producir una pérdida en el almacenamiento de energía celular (falla energética primaria) sobreviene la muerte temprana de una primera subpoblación celular por necrosis. Esto trae consigo la liberación de enzimas intracelulares al plasma. ${ }^{26-28}$ Enseguida continúa una fase de muerte celular tardía, en la que ocurre la muerte de una segunda subpoblación celular en forma progresiva. Esta subpoblación fue expuesta a un daño letal durante el episodio hipóxico-isquémico, pero sin que este alcanzara la intensidad suficiente para ocasionar la muerte inmediata (por ejemplo: edema citotóxico, daño mitocondrial, acumulación de excitotoxinas, acumulación de radicales libres, etc.). ${ }^{26-29}$

Lo anterior conduce a la muerte celular por apoptosis o necrosis en el lapso de horas a días posteriores al evento inicial. ${ }^{26-29}$ La liberación continua de enzimas intracelulares a la circulación debido a la necrosis de las células dañadas en forma irreversible explica la tendencia ascendente en los valores séricos de enzimas intracelulares que se observa en los pacientes con el evento asfíctico, pero no en los controles. Lo anterior se explica debido a que estos últimos no tuvieron muerte celular retardada en un grado notable y, por ello, se observa un aclaramiento enzimático en las horas subsecuentes al nacimiento.

Debido a que la lactato deshidrogenasa es una enzima distribuida en forma generalizada en prácticamente todas las células del organismo, su medición en plasma es un marcador sumamente sensible de daño celular.

En esta serie se demostró que la asociación entre el ascenso progresivo de la lactato deshidrogenasa (más de $0.33 \%$ /hora respecto del valor inicial en este estudio) persiste al menos durante las primeras 12 horas en todos los casos estudiados. Se obtiene mayor sensibilidad y especificidad si se compara el cambio en los valores séricos de lactato deshidrogenasa de muestras obtenidas con al menos 12 horas de diferencia entre ellas (0-12 horas).

También fue posible demostrar una asociación significativa en cualquiera de las muestras separadas por 6 horas ( 0 y 6 horas, 6 y 12 horas). Esta propiedad de la lactato deshidrogenasa la hace un marcador sumamente atractivo para la evaluación de la asfixia neonatal. Su empleo permitiría sustentar el diagnóstico de asfixia neonatal aun en ausencia de gasometría inicial (por ejemplo, pacientes nacidos fuera del medio hospitalario). Si estos hallazgos se confirman es posible que pueda incrementarse la proporción de neonatos con diagnóstico de probable asfixia que pudieran considerarse aptos para recibir tratamiento neuroprotector (en particular hipotermia terapéutica).

El escaso número de casos y controles es la principal limitante de este estudio; por ello resulta difícil la generalización y extrapolación de los resultados obtenidos. A pesar de ello la 
asociación observada entre los cambios con respecto al tiempo de vida extrauterina en las concentraciones séricas de lactato deshidrogenasa con la encefalopatía hipóxico-isquémica puede ser relevante en la práctica clínica, por lo que consideramos que esta variable debería ser tomada en cuenta en investigaciones posteriores.

\section{CONCLUSIONES}

Las concentraciones séricas de lactato deshidrogenasa y, en especial, la variación de estas en las primeras 12 horas de vida extrauterina tienen una asociación significativa con encefalopatía hipóxico-isquémica. Antes de trasladar los resultados a la práctica clínica se requieren más estudios que validen esta asociación.

\section{REFERENCIAS}

1. Beken S, Aydın B, Dilli D, Erol S, Zenciroğlu A, Okumuş N. Can biochemical markers predict the severity of hypoxic-ischemic encephalopathy? Turk J Pediatr. 2014 Feb;56(1):62-8.

2. Antonucci R, Porcella A, Pilloni MD. Perinatal asphyxia in the term newborn. J Pediatr Neonatal Individ Med JPNIM. 2014 Oct 21;3(2): e030269.

3. Nelson KB, Leviton A. How much of neonatal encephalopathy is due to birth asphyxia? Am J Dis Child 1960. 1991 Nov;145(11):1325-31.

4. Lawn JE, et al. Four million neonatal deaths: counting and attribution of cause of death. Paediatr Perinat Epidemiol. 2008 Sep;22(5):410-6. https://doi.org/10.1111/j.13653016.2008.00960.x

5. García-Alix A, et al. Neonatal hypoxic-ischemic encephalopathy: Incidence and prevalence in the first decade of the 21st century. An Pediatr Barc Spain 2003. 2009 Oct;71(4):319-26. DOI: 10.1016/j.anpedi.2009.07.021

6. Hayakawa $\mathrm{M}$, et al. Incidence and prediction of outcome in hypoxic-ischemic encephalopathy in Japan. Pediatr Int Off J Jpn Pediatr Soc. 2014 Apr;56(2):215-21. https://doi. org/10.1111/ped.12233

7. Bryce J, et al. WHO estimates of the causes of death in children. Lancet Lond Engl. 2005 Apr 26;365(9465):1147-52. https://doi.org/10.1016/S0140-6736(05)71877-8

8. Lindström $\mathrm{K}$, et al. Moderate neonatal encephalopathy: pre- and perinatal risk factors and long-term outcome. Obstetric Anesthesia Digest. 2008 Dec; 87(5):503-9. doi: 10.1097/01.aoa.0000337914.86125.54

9. Fernández Cantón S, Hernández Martínez AM, Viguri Uribe R. Mortalidad de la población de menores de cinco años en México durante 2011. Bol Med Hosp Infant Mex. 2013;70(1):66-69.

10. Castañeda-Casale G, Márquez-González H, RodríguezReyes ER. Mortalidad perinatal en un hospital de segundo nivel de atención. Rev Med Inst Mex Seguro Soc. 2010;48(3):237-42.

11. Sierra MTM, Lozano R, Santos JI. Mortalidad perinatal por asfixia en México: problema prioritario de salud pública por resolver. Bol Med Hosp Infant Mex. 2005;62(5):375-83.

12. Nelson KB, Bingham P, Edwards EM, Horbar JD, Kenny MJ, Inder T, et al. Antecedents of neonatal encephalopathy in the Vermont Oxford Network Encephalopathy Registry. Pediatrics. 2012 Nov;130(5):878-86.

13. Martinez-Biarge M, Diez-Sebastian J, Wusthoff CJ, Mercuri E, Cowan FM. Antepartum and intrapartum factors preceding neonatal hypoxic-ischemic encephalopathy. Pediatrics. 2013 Oct;132(4): e952-959.

14. Karlsson M, et al. Lactate dehydrogenase predicts hypoxic ischemic encephalopathy in newborn infants: a preliminary study. Acta Paediatr Oslo Nor. 2010 Aug;99(8):1139-44. https://doi.org/10.1111/j.1651-2227.2010.01802.x

15. Ramu D, Madapura.V P, Sabapathy S, Rajendra N. Study of dynamics of lactate dehydrogenase and hepatic enzymes activity following perinatal asphyxia in full term neonates. Pediatr Rev Int J Pediatr Res [Internet]. 2016 Sep 30 [cited 2018 Mar 1];3(09). http://medresearch.in/index.php/IJPR/ article/view/835

16. Karlsson $M$, et al. Dynamics of hepatic enzyme activity following birth asphyxia. Acta Paediatr Oslo Nor 1992. 2006 Nov;95(11):1405-11. https://doi. org/10.1080/08035250600693488

17. Magalhães M, Rodrigues FPM, Chopard MRT, Melo VC de A, Melhado A, Oliveira I, et al. Neuroprotective body hypothermia among newborns with hypoxic ischemic encephalopathy: three-year experience in a tertiary university hospital. A retrospective observational study. Sao Paulo Med J. 2015 Aug;133(4):314-9. http://dx.doi. org/10.1590/1516-3180.2013.7740026

18. Azzopardi DV, Strohm B, Edwards AD, Dyet L, Halliday HL, Juszczak E, et al. Moderate hypothermia to treat perinatal asphyxial encephalopathy. N Engl J Med. 2009 Oct 1;361(14):1349-58. DOI: 10.1056/NEJMoa0900854

19. Shankaran S, Laptook AR, Ehrenkranz RA, Tyson JE, McDonald SA, Donovan EF, et al. Whole-Body Hypothermia for Neonates with Hypoxic-Ischemic Encephalopathy. N Engl J Med. 2005 Oct 13;353(15):1574-84. DOI: 10.1056/ NEJMcps050929

20. Edwards $A D$, Brocklehurst $P$, Gunn AJ, Halliday H, Juszczak $E$, Levene $M$, et al. Neurological outcomes at 18 months of age after moderate hypothermia for perinatal hypoxic ischemic encephalopathy: synthesis and meta-analysis of trial data. BMJ. 2010 Feb 9;340:c363. https://doi. org/10.1136/bmj.c363

21. Karlsson M, Tooley JR, Satas S, Hobbs CE, Chakkarapani E, Stone J, et al. Delayed hypothermia as selective head cool- 
ing or whole-body cooling does not protect brain or body in newborn pig subjected to hypoxia-ischemia. Pediatr Res. 2008 Jul;64(1):74-8.

22. Jacobs SE, Berg M, Hunt R, Tarnow-Mordi WO, Inder TE, Davis PG. Cooling for newborns with hypoxic ischemic encephalopathy. Cochrane Database Syst Rev. 2013 Jan 31;(1):CD003311.

23. Queensland Clinical Guidelines. Hypoxic-ischaemic encephalopathy (HIE). Australia: Queensland Health, 2017;1-26.

24. Cruz-Cauich J, Lagunes-Cordoba R. El lactato en sangre como prueba diagnóstica de encefalopatía isquémica en neonatos. Rev Invest Cien Sal. 2012;7(2):22-8.

25. Wiberg N, Källén K, Herbst A, Olofsson P. Relation between umbilical cord blood $\mathrm{pH}$, base deficit, lactate, 5-minute Apgar score and development of hypoxic isch- emic encephalopathy. Acta Obstet Gynecol Scand. 2010 Oct;89(10):1263-9.

26. Jaeschke $\mathrm{H}$, Lemasters JJ. Apoptosis versus oncotic necrosis in hepatic ischemia/reperfusion injury. Gastroenterology. 2003 Oct;125(4):1246-57.

27. Mehmet H, Yue X, Penrice J, Cady E, Wyatt JC, Sarraf C, et al. Relation of impaired energy metabolism to apoptosis and necrosis following transient cerebral hypoxia-ischaemia. Cell Death Differ. 1998 Apr;5(4):321-9.

28. Yue X, Mehmet H, Penrice J, Cooper C, Cady E, Wyatt JS, et al. Apoptosis and necrosis in the newborn piglet brain following transient cerebral hypoxia-ischaemia. Neuropathol Appl Neurobiol. 1997 Feb;23(1):16-25.

29. Inder TE, Volpe JJ. Mechanisms of perinatal brain injury. Semin Neonatol SN. 2000 Feb;5(1):3-16. 